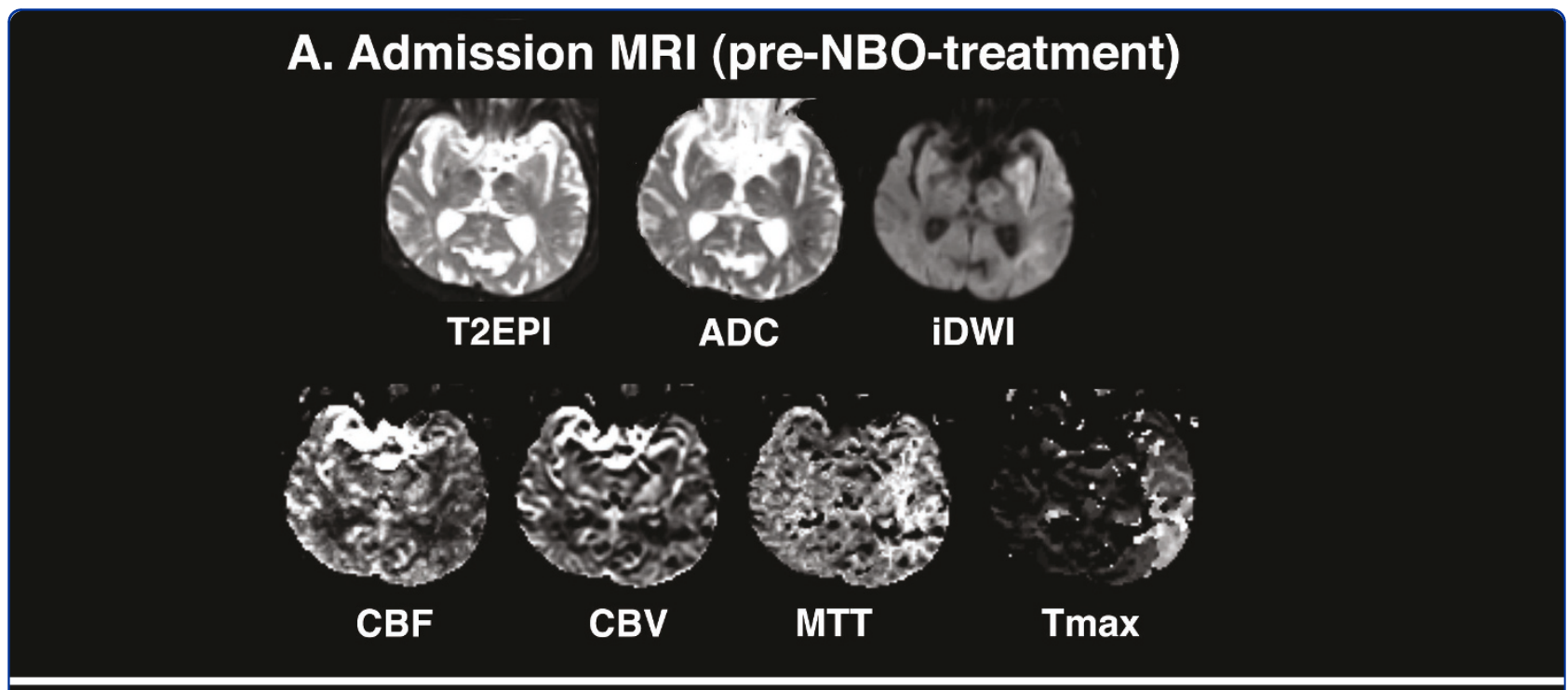

B. Predicted Lesion Volumes and Their Differences

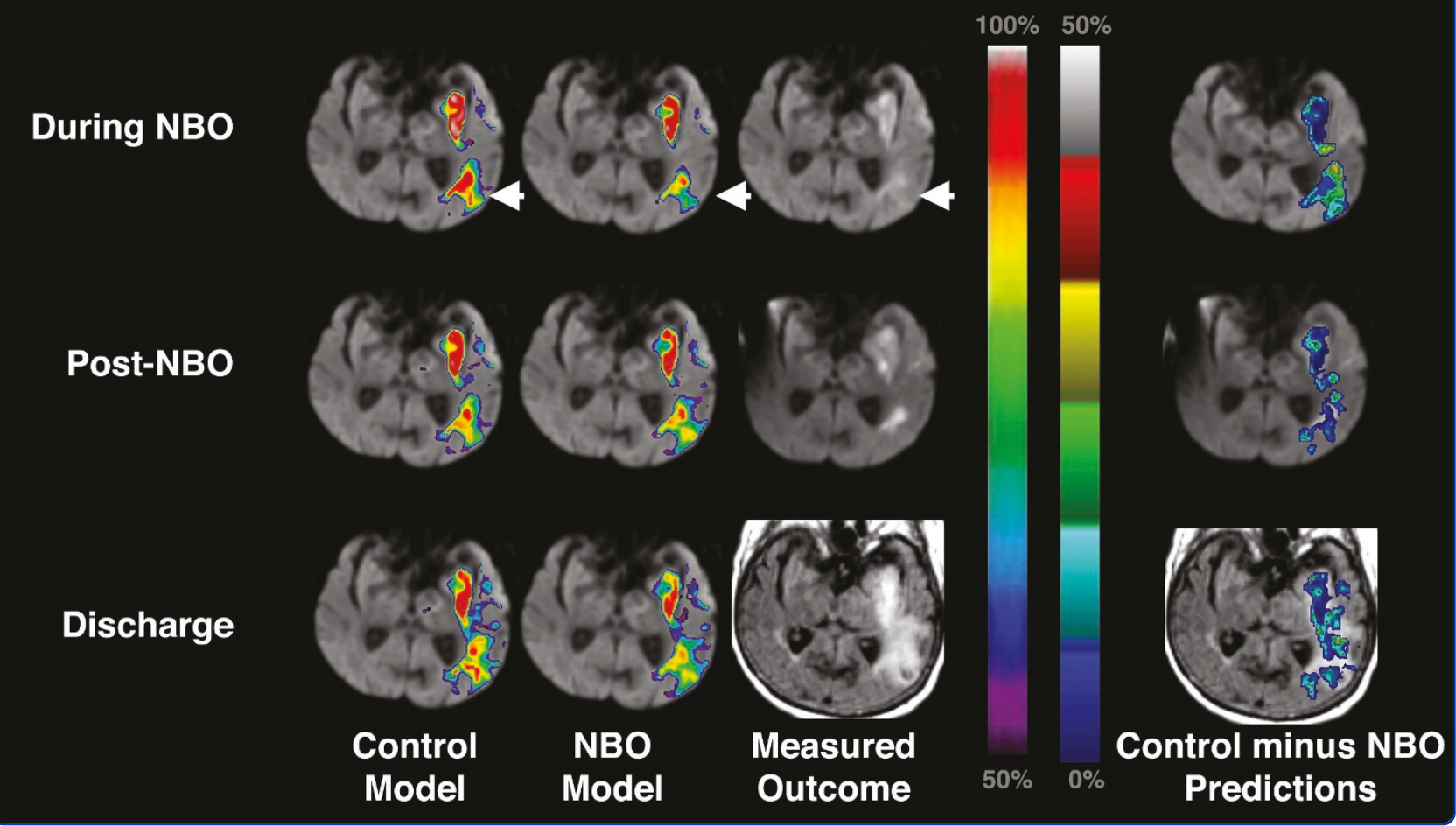

Evaluating effects of normobaric oxygen therapy in acute stroke with MRI-based predictive models Wu et al. 


\title{
Evaluating effects of normobaric oxygen therapy in acute stroke with MRI-based predictive models
}

\author{
Ona Wu', Thomas Benner ${ }^{1}$, Luca Roccatagliata ${ }^{3}$, Mingwang Zhu', Pamela W Schaefer ${ }^{3}$, Alma Gregory Sorensen ${ }^{1}$ \\ and Aneesh B Singhal ${ }^{2 *}$
}

\begin{abstract}
Background: Voxel-based algorithms using acute multiparametric-MRI data have been shown to accurately predict tissue outcome after stroke. We explored the potential of MRI-based predictive algorithms to objectively assess the effects of normobaric oxygen therapy (NBO), an investigational stroke treatment, using data from a pilot study of NBO in acute stroke.
\end{abstract}

Methods: The pilot study of NBO enrolled 11 patients randomized to NBO administered for 8 hours, and 8 Control patients who received room-air. Serial MRIs were obtained at admission, during gas therapy, post-therapy, and predischarge. Diffusion/perfusion MRI data acquired at admission (pre-therapy) was used in generalized linear models to predict the risk of lesion growth at subsequent time points for both treatment scenarios: NBO or Control.

Results: Lesion volume sizes 'during NBO therapy' predicted by Control-models were significantly larger $(P=0.007)$ than those predicted by NBO models, suggesting that ischemic lesion growth is attenuated during NBO treatment. No significant difference was found between the predicted lesion volumes at later time-points. NBO-treated patients, despite showing larger lesion volumes on Control-models than NBO-models, tended to have reduced lesion growth.

Conclusions: This study shows that NBO has therapeutic potential in acute ischemic stroke, and demonstrates the feasibility of using MRI-based algorithms to evaluate novel treatments in early-phase clinical trials.

Keywords: Stroke, Clinical Trial, Oxygen Therapy, Neuroprotection, MRI multiparametric models

\section{Background}

MRI voxel-based predictive algorithms based on admission MRI datasets including diffusion-weighted imaging (DWI) and perfusion-weighted imaging (PWI), have been shown to accurately predict tissue outcomes in acute stroke patients $[1,2]$ and animal stroke models [3-6]. In the acute stroke setting, these models can be used to predict the patient's response to various treatment options, enable better treatment selection, and thereby improve patient outcome. Furthermore, MRIbased algorithms have potential to be used in earlyphase clinical trials to assess the efficacy and toxicity of investigational therapies by providing an estimate of tissue likely to infarct without intervention, and comparing

\footnotetext{
* Correspondence: asinghal@partners.org

${ }^{2}$ Department of Neurology, Massachusetts General Hospital, 55 Fruit Street, Boston MA 02114, USA

Full list of author information is available at the end of the article
}

this predicted outcome to the actual tissue status observed post-treatment. Such algorithms have been successfully used to demonstrate therapeutic efficacy of recombinant tissue plasminogen activator (rt-PA) in animal [3] and human [7] studies. In these two studies, the group given rt-PA had smaller actual lesion volumes than was predicted using natural history models.

In this study, we extend our prior research by developing models under treatment and control conditions in order to predict tissue outcome after normobaric oxygen therapy (NBO), a promising investigational acute stroke treatment [8-17]. We analyzed data from a prospective randomized pilot study of normobaric oxygen therapy (NBO) [18-20]. In the pilot study [18], NBO transiently improved clinical deficits and reduced DWI lesions in stroke patients with mismatches in lesion volumes on DWI and PWI. These effects were most evident during therapy, suggesting that NBO may slow down the 
process of ischemic necrosis and hence be a simple strategy to extend the narrow time window for stroke thrombolysis. In the present study we compared predicted tissue outcome on a voxel-wise basis under the two treatment strategies, Room-air and NBO. We postulated that differences between the expected outcomes of these models will objectively validate prior observations and provide insights into NBO's mechanisms.

\section{Methods \\ Patients}

This study was approved by our Hospital's Human Research Committee (approval number 2001P001176). The NBO pilot study inclusion and exclusion criteria, DWI and PWI imaging methods, and clinical and volumetric results have been published [18-20]. Data from three additional randomized patients (2 treated with $\mathrm{NBO}$, one with room air), obtained after the original report, were incorporated in this analysis. Briefly, patients were eligible if they presented $<12 \mathrm{~h}$ after witnessed stroke onset or $<15 \mathrm{~h}$ after last seen neurologically intact, and had a $>20 \%$ visually-estimated mismatch in acute DWI and PWI lesion volumes. After obtaining informed consent, patients were randomized to room air (Control, $\mathrm{n}=8$ ) or NBO (high-flow oxygen delivered via a facemask for 8 hours, $\mathrm{n}=11)$. All patients received MRIs upon admission (pre-treatment), $4 \mathrm{~h}$ later (during treatment), $24 \mathrm{~h}$ (post-treatment) and before discharge. One patient from each group was excluded due to hyperacute post-ischemic hemorrhage [21]. Another Control patient was excluded due to imaging artifacts. Reperfusion, defined as recanalization of a previously occluded artery on MR-angiography (MRA) or $>50 \%$ decrease in mean transit time (MTT) lesion volume, was measured at $4 \mathrm{~h}$ and $24 \mathrm{~h}$.

\section{Image acquisition}

DWI was acquired with b-value $=0 \mathrm{~s} / \mathrm{mm}^{2}$ and six diffusion-weighted images with $\mathrm{b}$-value $=1000 \mathrm{~s} / \mathrm{mm}^{2}$. Apparent diffusion coefficient (ADC) maps were calculated as the negative slope of the linear regression fit of the log DWI images versus their b-values. The isotropic DWI map (iDWI) was calculated as the geometric mean of the diffusion-weighted images. The b-value $=0 \mathrm{~s} /$ $\mathrm{mm}^{2}$ acquisition was used as the $\mathrm{T}_{2}$-weighted image $\left(\mathrm{T}_{2} \mathrm{WI}\right)$. Non-uniformity correction was performed on both the iDWI and the $\mathrm{T}_{2} \mathrm{WI}$ data sets [22]. PWI was performed by acquiring dynamic susceptibility contrastweighted images during the first pass of a bolus of highmagnetic susceptibility contrast agent. Cerebral blood flow $(\mathrm{CBF})$, cerebral blood volume (CBV), mean transit time (MTT), and delay maps (Tmax) maps were calculated using local oscillation-index regularized singular value decomposition with a block-circulant matrix [23] for deconvolving concentration-time curves with an automatically selected arterial input function [24].

\section{GLM development}

Details regarding the development of generalized linear models (GLM) for predicting tissue infarction have been published [7]. Briefly, tissue outcome is modeled as a binary variable, with 1 representing lesioned tissue and 0 non-lesioned tissue. The probability of tissue lesion development $(P)$ can then be represented by the logistic function, $\mathrm{P}=\frac{1}{1+\mathrm{e}^{-\eta(\mathbf{x})}}$ (Equation 1), for which $\eta(\mathrm{x})$ is a linear function of its input parameters, $\eta(\mathbf{x})=\beta^{\mathrm{T}} \mathbf{x}+\alpha$ (Equation 2) and $\beta$ is the vector of weighting coefficients and $\alpha$ is the bias or intercept term for the GLM. For this study, the input vector, $\mathbf{x}$, consisted of the acute $\mathrm{T}_{2} \mathrm{WI}, \mathrm{ADC}$, iDWI, CBF, CBV, MTT and Tmax maps. All images were co-registered using semi-automated image registration software to one another (FLIRT [25]) and to a probabilistic brain atlas [26] (MNI Autoreg [27]), and then normalized with respect to mean values measured in the normal contralateral white matter using previously described techniques [7]. Training regions consisted of tissue lesions delineated by a neuroradiologist as regions of hyperintensities on $4 \mathrm{~h}$ or $24 \mathrm{~h}$ DWIs or before discharge MRIs (performed approximately 1week later), and non-lesions defined as remaining ipsilateral hemisphere tissue not demonstrating artifacts on admission MRI. Coefficients of the GLM were calculated using an iterative re-weighted least squares algorithm ( $R$ [28]) and then applied to admission MRI to produce lesion risk maps for each patient. For all models, the bootstrapped estimate [29] of the mean of the GLM coefficients were used, with care taken that an equal number of lesion and non-lesion samples were used for each iteration to compensate for imbalanced training data which can lead to models weighted towards predicting the majority class, i.e. non-lesion tissue [30].

Two sets of models were created to predict lesion growth at each of the three subsequent time points: $4 \mathrm{~h}$ (during treatment), $24 \mathrm{~h}$ (post-treatment) and Discharge. Control-models were trained using all room-air treated patients $(n=6)$. NBO-models were trained on oxygentreated patients $(n=10)$. Lesion risk maps for the NBOtreated cohort were calculated using both types of models. To avoid bias from training and testing on the same data, jack-knifing or leave-one-out approach was used to evaluate the performance of the NBO-models [29]. The coefficients for the models were tested for significance and compared to one another (Z-tests).

\section{Data analysis}

All analyses were performed on co-registered datasets. Manually outlined lesions at $4 \mathrm{~h}, 24 \mathrm{~h}$ and Discharge 
were used to train the GLMs and to define the measured lesion volume (MLV) at each successive time point. Lesion Change, defined as the ratio of the MLV at each time point to the admission DWI lesion volume, was compared between the $\mathrm{NBO}$ and control groups (unpaired Student $t$-test). The predicted lesion volume (PLV) was defined as tissue where GLM-predicted lesion risk was greater than a threshold of $50 \%$. A threshold of $50 \%$ was selected since the models were designed to produce the optimal operating point at this cutoff by using an equal number of lesion and non-lesion voxels as part of the bootstrapped training. PLV at each time point using each model PLV $_{\text {Control }}$ and $\mathrm{PLV}_{\mathrm{NBO}}$ ) were calculated and compared. Regional analysis was performed comparing $\mathrm{T}_{2} \mathrm{WI}, \mathrm{ADC}$, DWI, CBF, CBV, MTT and Tmax in areas where the PLV of both models matched with areas where $\mathrm{PLV}_{\text {Control }}$ were greater than areas of $\mathrm{PLV}_{\mathrm{NBO}}$. Correlations between the two models were calculated (Pearson's product-moment) at each time point. Correlations were also performed between ratios of $P L V_{\text {Control }}$ to $P L V_{N B O}$ with respect to Lesion Change to predict the expected responsiveness of individual patients to NBO.

Region of interest (ROI) analysis were performed in tissue that was correctly predicted to become lesioned (true positive, $\mathrm{TP}$ ) by Control-models $\left(\mathrm{TP}_{\text {Control }}\right)$ or NBO-models $\left(\mathrm{TP}_{\mathrm{NBO}}\right)$, in tissue that was falsely predicted to become lesioned (false positive, FP) using the
Control-model ( $\left.\mathrm{FP}_{\text {Control }}\right)$ or the NBO-model $\left(\mathrm{FP}_{\mathrm{NBO}}\right)$, in tissue correctly predicted to not become a lesion (true negative, $\mathrm{TN})$ for Control-models $\left(\mathrm{TN}_{\mathrm{Control}}\right)$ or $\mathrm{NBO}$-models $\left(\mathrm{TN}_{\mathrm{NBO}}\right)$, and in tissue incorrectly predicted to not become lesioned (false negative, FN) for Control-models $\left(\mathrm{FN}_{\text {Control }}\right)$ or NBO-models $\left(\mathrm{FN}_{\mathrm{NBO}}\right)$. Mean predicted lesion risk in each region was calculated and compared (ANOVA with post-hoc Tukey HSD test) for each model. All statistical comparisons used onesided Wilcoxon-tests, unless otherwise noted. $\mathrm{P}<0.05$ was considered significant for all analyses, which were limited to slices that had valid data for all co-registered input image sets.

\section{Results}

Table 1 shows patient characteristics. Measured lesion volumes were not significantly different between groups at any time point. NBO-treated patients had larger admission DWI lesion volumes and consequently tended to have larger absolute lesion volumes at all subsequent time points. An analysis of lesion volume change from before (baseline) to during treatment $(4 \mathrm{~h})$ showed significant reduction in DWI lesion size in NBO-treated patients and an increase in Control patients, consistent with previous reports [18]. At the other time points, lesion expansion was observed in both groups, but to a lesser extent in the NBO-treated group.

Table 1 Clinical and imaging characteristics

\begin{tabular}{|c|c|c|}
\hline & Control-treated $(n=6)$ & NBO-treated $(n=10)$ \\
\hline & Mean \pm SD (Median) & Mean \pm SD (Median) \\
\hline Age (y) & $71 \pm 18(71)$ & $67 \pm 16(70)$ \\
\hline Female & $4(67 \%)$ & $5(50 \%)$ \\
\hline \multicolumn{3}{|l|}{ Time Intervals } \\
\hline Symptom onset to admission MRI (h) & $4.4 \pm 2.0(4.4)$ & $7.3 \pm 4.5(5.9)$ \\
\hline Admission-to-treatment MRI (h) & $5.0 \pm 1.4(4.8)$ & $4.3 \pm 1.5(3.9)$ \\
\hline Admission-to-post-treatment MRI (h) & $24.8 \pm 1.9(24.8)$ & $24.4 \pm 1.5(24.2)$ \\
\hline Admission-to-discharge MRI (d) & $6.7 \pm 1.9(6.6)$ & $6.0 \pm 1.4(5.8)$ \\
\hline Acute NIHSSS & $11(9-12)$ & $14(9-18)$ \\
\hline \multicolumn{3}{|l|}{ Reperfusion } \\
\hline Admission-to-treatment & $1(17 \%)$ & 0 \\
\hline Treatment to post-treatment & 0 & $5(50 \%)$ \\
\hline \multicolumn{3}{|l|}{ Measured Lesion Volume $\left(\mathrm{cm}^{3}\right)$} \\
\hline Admission DWI & $27 \pm 44(8) \mathrm{cm}^{3}$ & $37 \pm 24(30) \mathrm{cm}^{3}$ \\
\hline $4 \mathrm{~h} \mathrm{DWl}$ & $28 \pm 34(15) \mathrm{cm}^{3}$ & $35 \pm 25(27) \mathrm{cm}^{3}$ \\
\hline $24 \mathrm{~h} \mathrm{DWl}$ & $32 \pm 39(15) \mathrm{cm}^{3}$ & $49 \pm 37(43) \mathrm{cm}^{3}$ \\
\hline Discharge & $41 \pm 48(23) \mathrm{cm}^{3}$ & $66 \pm 42(53) \mathrm{cm}^{3}$ \\
\hline \multicolumn{3}{|l|}{ Lesion Change (\%) } \\
\hline $4 \mathrm{~h}^{*}$ & $153 \pm 96(134) \%$ & $90 \pm 22(90) \%$ \\
\hline $24 \mathrm{~h}$ & $186 \pm 142(148) \%$ & $129 \pm 29(134) \%$ \\
\hline Discharge & $223 \pm 91(203) \%$ & $192 \pm 81(178) \%$ \\
\hline
\end{tabular}

*P $=0.036$ Control-group vs NBO-group 
Control- and NBO-models were trained to predict lesion development on a voxel-wise basis at $4 \mathrm{~h}, 24 \mathrm{~h}$ and Discharge. The coefficients for the models using all patient data in each respective cohort are shown in Table 2. These coefficients represent the relative importance of each covariate on the likelihood of lesion development. There were significant differences $(\mathrm{P}<0.05)$ between the coefficients as a function of treatment type (i.e. NBO-model or Control-model) or time of lesion development (i.e. 4 h, 24 h, or Discharge). The coefficients changed over time, with the iDWI coefficient having less import for predicting outcome at later timepoints for both NBO- and Control-models.

Examples of predicted lesion development using the two models for NBO-treated patients are shown in Figures 1 and 2, along with the voxel-wise differences. Note the greatest differences between Control and NBO models in the predicted risk of lesion development are observed at $4 \mathrm{~h}$.

As compared to lesion volumes predicted by NBOmodels, the Control-model predicted significantly larger $(\mathrm{P}=0.007)$ expected lesion volumes in the NBO-treated group at $4 \mathrm{~h}\left(52 \pm 30\right.$ vs. $\left.46 \pm 31 \mathrm{~cm}^{3}\right)$, but not at $24 \mathrm{~h}$ $\left(58 \pm 33\right.$ vs. $\left.59 \pm 36 \mathrm{~cm}^{3}\right)$ or Discharge $(74 \pm 36$ vs. 75 $\pm 38 \mathrm{~cm}^{3}$ ). The mean \pm SD difference between $\mathrm{PLV}_{\text {Con- }}$ trol and PLV $\mathrm{NBO}_{\mathrm{NBO}}$ for $4 \mathrm{~h}, 24 \mathrm{~h}$, and Discharge were $5.3 \pm$ $6.4,-1.0 \pm 6.8$, and $-0.7 \pm 5.7 \mathrm{~cm}^{3}$. In regions where $\mathrm{PLV}_{\text {Control }}$ was greater than $\mathrm{PLV}_{\mathrm{NBO}}, \mathrm{T}_{2} \mathrm{WI}, \mathrm{ADC}$, DWI, $\mathrm{CBF}, \mathrm{CBV}$ and Tmax were significantly different $(\mathrm{P}<$ 0.05) from regions where PLV $V_{\text {Control }}$ matched $P L V_{N B O}$ for $4 \mathrm{~h}$ predictions. For the $24 \mathrm{~h}$ predictions, significant differences $(\mathrm{P}<0.05)$ were found for DWI, CBF, CBV, and Tmax. For the Discharge predictions, significant differences $(P<0.05)$ were found only for $T_{2} W I$, DWI, $\mathrm{CBF}, \mathrm{CBV}$, and Tmax. $\mathrm{ADC}, \mathrm{CBF}, \mathrm{CBV}$ and $\mathrm{CBF}$ were more reduced, while $\mathrm{T}_{2}$ WI, DWI and Tmax were higher in the matched regions where both models predicted lesion development compared to regions of discordance between the models.

ROI analysis shows that lesion risk is greatest in TP than in FP for both models at all time points (Figure $3 \mathrm{~A}-\mathrm{C}$ ). Lesion risk in these regions is also shown to significantly change over time.

For all time-points, the predicted lesion volumes were significantly correlated with the measured (manually outlined) lesion volumes for both the Control-models ( $4 \mathrm{~h}$ : $\mathrm{R}$ $=0.94 \mathrm{P}<0.0001 ; 24 \mathrm{~h}: \mathrm{R}=0.95 \mathrm{P}<0.0001$; Discharge: $\mathrm{R}$ $=0.88 \mathrm{P}<0.001)$ and the NBO-treatment models $(4 \mathrm{~h}: \mathrm{R}$ $=0.97 \mathrm{P}<0.0001 ; 24$ h: $\mathrm{R}=0.97 \mathrm{P}<0.0001$; Discharge: $\mathrm{R}$ $=0.83 \mathrm{P}<0.01)$. Lesion Change at $4 \mathrm{~h}$ had a tendency to be correlated with expected responsiveness to therapy, i.e. the ratio of $\mathrm{PLV}_{\text {Control }} / \mathrm{PLV}_{\mathrm{NBO}},(\mathrm{R}=-0.61 \mathrm{P}=0.06)$, but was poorly correlated at $24 h(R=-0.56 P=0.09)$ and at Discharge $(\mathrm{R}=0.52 \mathrm{P}=0.12)$ (Figure 4$)$. When DWI lesions were observed to shrink rather than expand at the $4 \mathrm{~h}$ time point, $\mathrm{PLV}_{\text {Control }} / \mathrm{PLV}_{\mathrm{NBO}}$ was $121 \pm 22 \%$.

\section{Discussion}

In this study, we used objective methods to validate prior observations showing the safety and efficacy of 8hour NBO in selected patients with acute ischemic stroke. Further, we show that paired analysis of expected outcomes under two therapeutic settings (control and $\mathrm{NBO}$ ) can be used to evaluate effects of novel therapeutic interventions. Using multivariate voxel-based algorithms, we can compare the expected outcome of each voxel of tissue (were the natural cascade of ischemic events to proceed unimpeded), versus their fate with therapy. Despite the imbalanced number of subjects or data in small pilot studies that occurs regardless of randomization, as was true for this investigation and reflected by the slightly larger DWI lesions in the NBOarm, we were still able to detect an alteration in the patient's lesion evolution from NBO-therapy.

Table 2 GLM Coefficients for the different models (Mean \pm SD) for predicting lesion development

\begin{tabular}{|c|c|c|c|c|c|c|c|c|}
\hline Models & Bias & $\mathrm{T}_{2} \mathrm{WI}$ & ADC & iDWI & $\mathrm{CBF}$ & CBV & MTT & Tmax \\
\hline \multicolumn{9}{|c|}{ Models Predicting $4 \mathrm{~h}$ Lesion Development (during treatment) } \\
\hline Control & $-11 \pm 1.4 \S$ & $4.0 \pm 1.0 \S$ & $-4.3 \pm 1.3 \S$ & $9.3 \pm 1.2 \S$ & $-0.9 \pm 0.2$ & $0.7 \pm 0.1$ & $0.5 \pm 0.2$ & $0.07 \pm 0.008 \S$ \\
\hline $\mathrm{NBO}$ & $-17 \pm 0.8 \S$ & $2.0 \pm 0.3 \S$ & $0.10 \pm 0.3^{*} \S$ & $11 \pm 0.5 \S$ & $-0.4 \pm 0.2^{*} \S$ & $-0.02 \pm 0.2^{*}$ & $0.9 \pm 0.2$ & $0.05 \pm 0.008 \S$ \\
\hline \multicolumn{9}{|c|}{ Models Predicting $24 \mathrm{~h}$ Lesion Development (post-treatment) } \\
\hline Control & $-3.1 \pm 1.0 \dagger$ & $8.1 \pm 0.8+$ & $-9.1 \pm 1.0 \dagger$ & $2.3 \pm 0.8+$ & $-0.7 \pm 0.1$ & $0.5 \pm 0.1$ & $0.5 \pm 0.1$ & $0.05 \pm 0.007 \dagger$ \\
\hline $\mathrm{NBO}$ & $-13 \pm 0.5+$ & $0.6 \pm 0.2+$ & $1.0 \pm 0.2+$ & $8.9 \pm 0.3 \dagger$ & $0.2 \pm 0.09+$ & $-0.2 \pm 0.08$ & $1.3 \pm 0.1$ & $0.1 \pm 0.006 t$ \\
\hline \multicolumn{9}{|c|}{ Models Predicting Discharge Lesion Development } \\
\hline Control & $-3.4 \pm 0.6+$ & $4.2 \pm 0.4 \S$ & $-5.1 \pm 0.5 \S$ & $2.7 \pm 0.5+$ & $-0.7 \pm 0.1$ & $0.5 \pm 0.08$ & $0.9 \pm 0.1 \S \dagger$ & $0.06 \pm 0.006$ \\
\hline $\mathrm{NBO}$ & $-8.8 \pm 0.3+\S$ & $0.3 \pm 0.1 \dagger$ & $0.7 \pm 0.2$ & $6.1 \pm 0.2+\S$ & $-0.2 \pm 0.09 * \S$ & $-0.06 \pm 0.07^{*}$ & $0.9 \pm 0.09 \S$ & $0.1 \pm 0.006+$ \\
\hline
\end{tabular}

*P $>0.05$ Non-significant coefficients

$+\mathrm{P}<0.05$ vs coefficients of $4 \mathrm{~h}$ models, $\S \mathrm{P}<0.05$ vs coefficients of $24 \mathrm{~h}$ models

Coefficients for Control- and NBO-models were significantly different $(P<0.05)$ with the exception of $T_{2}$ Wl, iDWI, CBF and Tmax for 4 h and MTT for 1 -week time points (displayed in light-gray). 


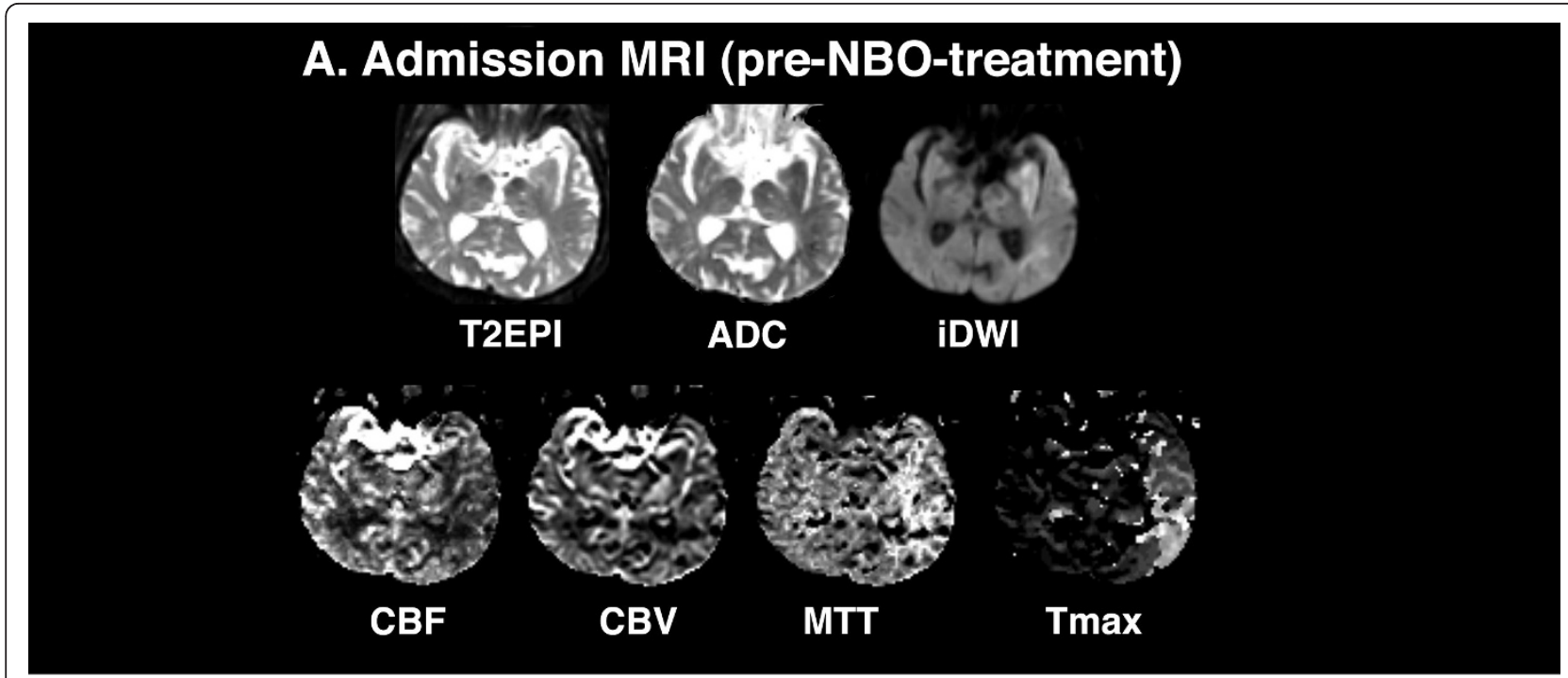

\section{B. Predicted Lesion Volumes and Their Differences}

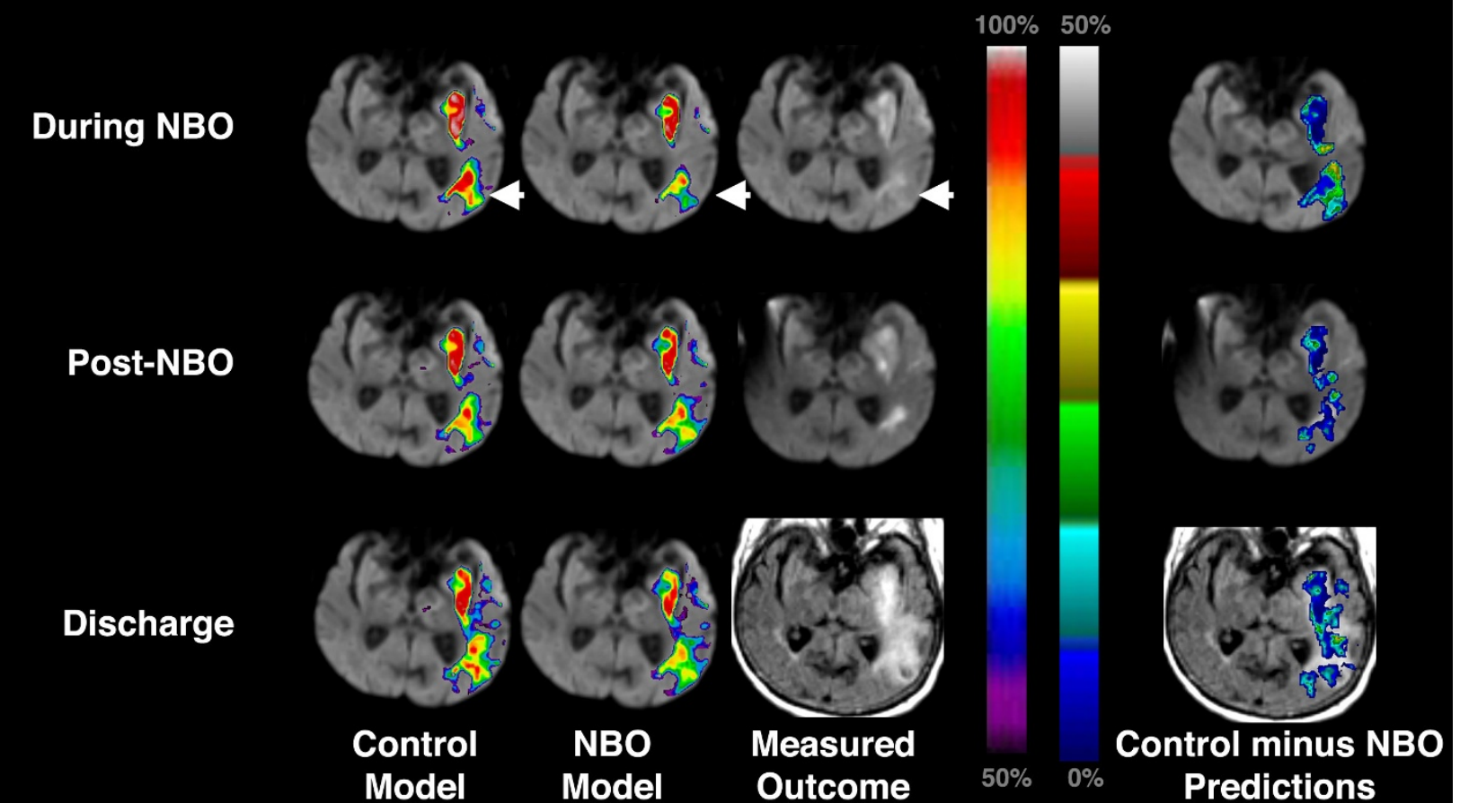

Figure 1 Example 1 of Predicted Lesion Volume Development. (A) Admission MRI dataset for a 79 year-old woman with stroke who was imaged 13 hours after she was last seen well and treated with NBO. (B) Corresponding GLM-predicted lesion risk maps (left panel) for Control and NBO-models at each subsequent time point of imaging, and overlay map (right panel) of differences between the two models showing ischemic tissue that is 'potentially salvageable' with NBO therapy. The GLM-predicted lesion volumes are asynthesis of data from the admission MRI only. In this patient, the risk of tissue infarction in DWI/PWI mismatch regions was predicted to increase over time. For clarity, only GLMpredicted lesion risk > 50\% are shown overlaid on acute DWI. Note that the amounts of tissue predicted to infarct at all time-points with the Control-models were greater than their NBO-model counterparts, with difference principally in the DWI/PWI mismatch region (arrowheads). In the difference maps ( $\mathrm{B}$, right panel), the color scale represents infarction risk reduction as a result of NBO-therapy (conversely, larger values represent greater likelihood of infarction if the patient was given Control-treatment).

In our NBO pilot study [18], oxygen therapy transiently improved clinical deficits and DWI lesion volumes in stroke patients presenting with mismatches in lesion volumes on DWI and PWI. This transient beneficial effect of NBO has also been shown in rodent stroke studies [10-17]. Similarly in this study we found that DWI 

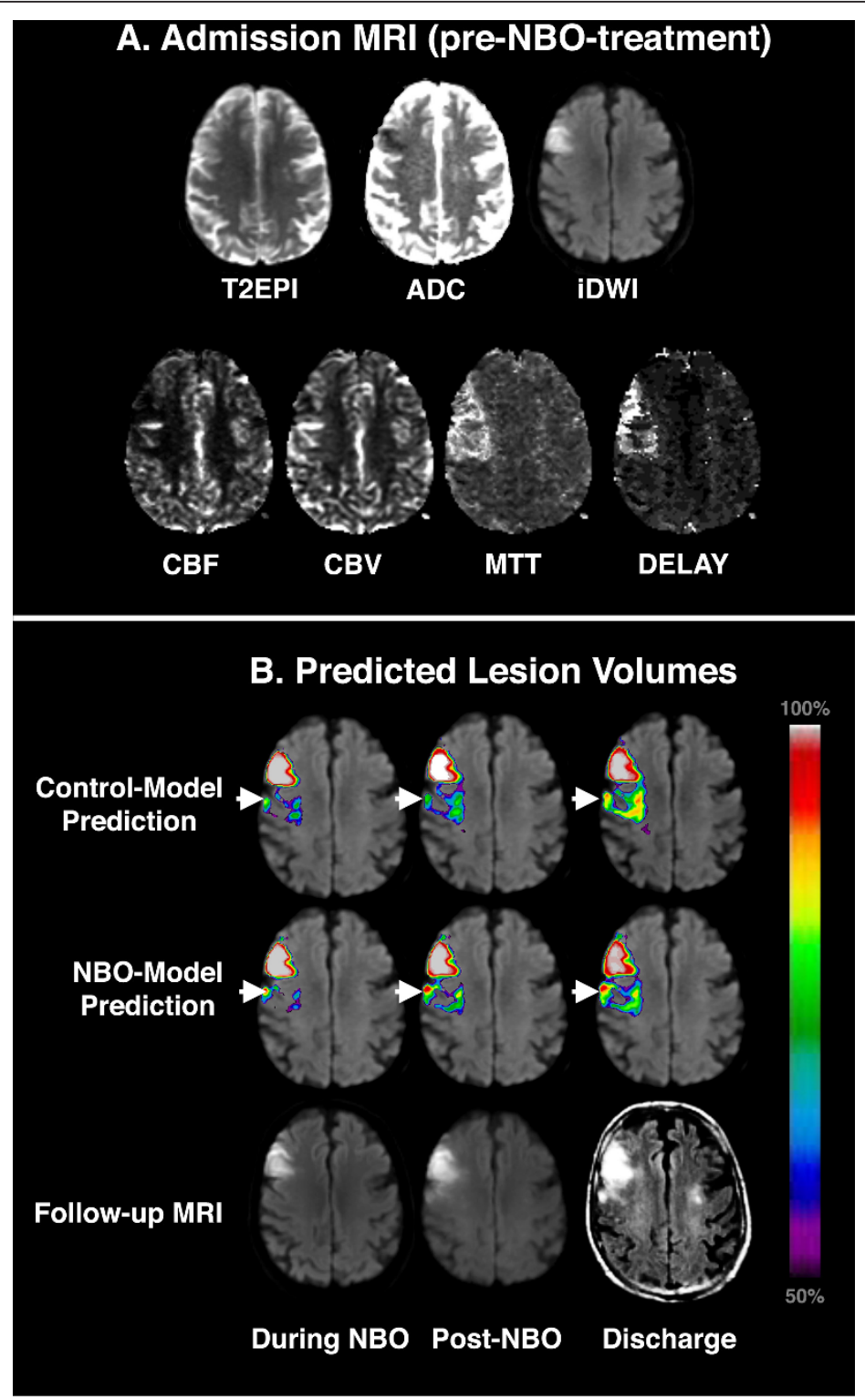

C. Infarction Risk Reduction

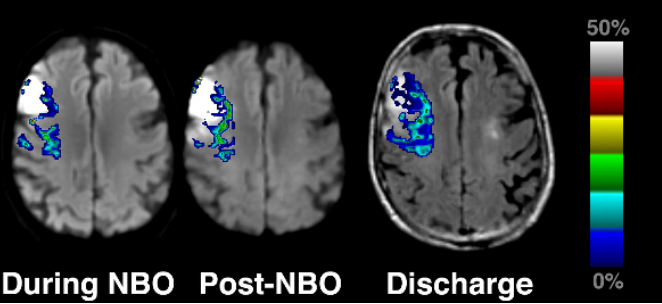

Figure 2 Example 2 of Predicted Lesion Volume Development. (A) Admission MRI dataset for a 64 year-old male patient imaged at 4.5 hours after stroke symptom onset who was treated with NBO. (B) Corresponding GLM-predicted lesion risk maps for Control and NBO-models at each subsequent time point of imaging, and (C) Overlay map of differences between the two models showing ischemic tissue that is 'potentially salvageable' with NBO therapy. The GLM-predicted lesion volumes are a synthesis of data from the admission MRI only. In this patient, the risk of tissue infarction in DWI/PWI mismatch regions was predicted to increase over time. For clarity, only GLM-predicted lesion risk $>50 \%$ are shown overlaid on acute DWI. Note that the amounts of tissue predicted to infarct at all time-points with the Control-models were greater than their NBO-model counterparts, with difference principally in the DWI/PWI mismatch region (arrowheads). In the difference maps (C), the color scale represents infarction risk reduction as a result of NBO-therapy (conversely, larger values represent greater likelihood of infarction if the patient was given Control-treatment). 


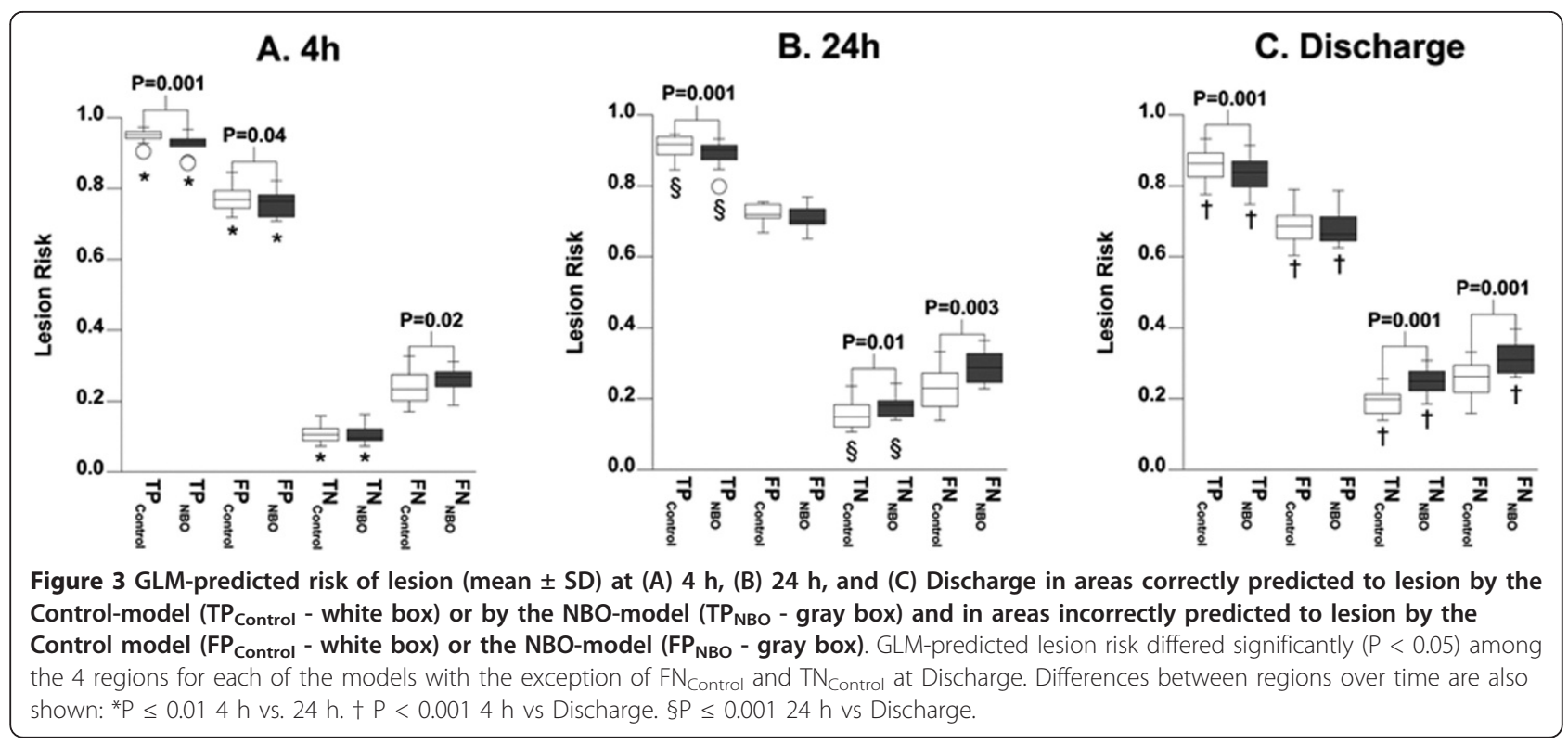

lesion volume growth with respect to baseline in the NBO-arm was reduced at $4 \mathrm{~h}$ compared to the Controlarm. The current study extends these change-from-baseline volumetric methods in individual modalities since combining multiple imaging modalities have been shown to more accurately predict tissue infarction than any currently existing individual technique $[1,2]$. Predictive modeling approaches also have the potential to test

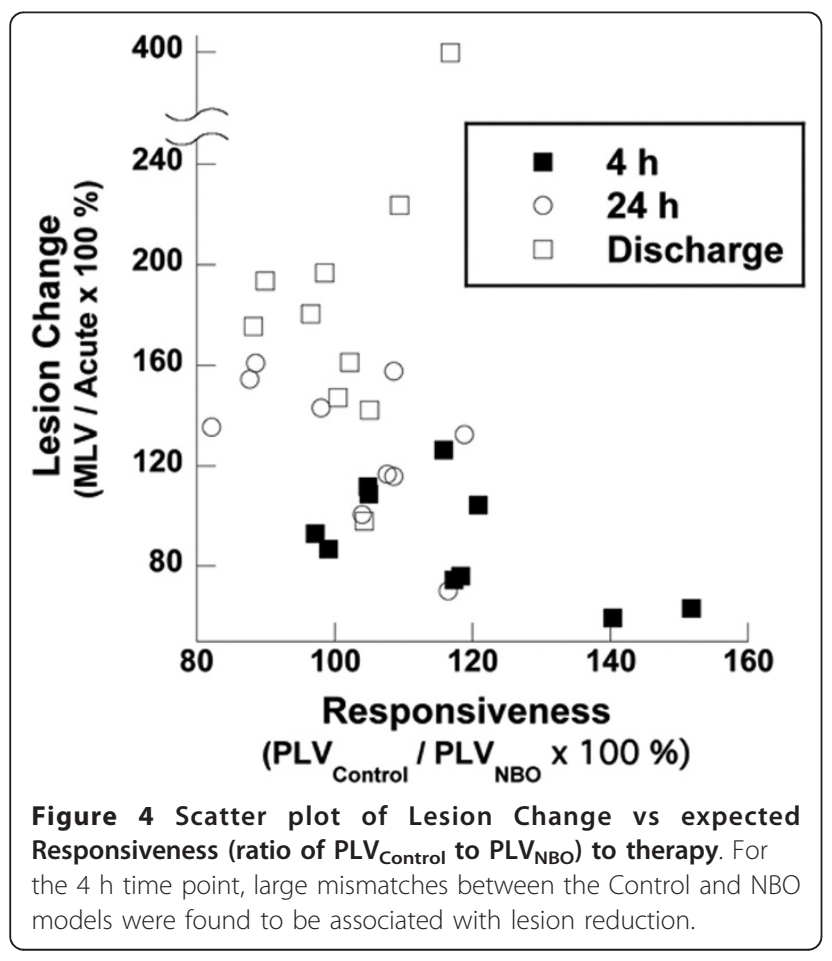

whether a new therapy worsens tissue outcome. A theoretical concern with oxygen therapy is an increased risk of generating toxic oxygen free radicals [31]. By showing that the predicted lesion volumes assuming NBO-treatment was not greater than those assuming room-airtreatment, our objective data provides evidence that NBO-therapy may not worsen tissue outcome at Discharge.

In this study, we extend our previous GLM modeling approach $[1,7]$, which were limited to "natural history models," by developing tissue-signature models that use pre-treatment data to predict expected tissue outcome if a novel therapy was to be given. Our results showed that during therapy $\mathrm{FP}_{\text {Control }}$ was significantly higher than $\mathrm{FP}_{\mathrm{NBO}}$, suggesting $\mathrm{NBO}$-treatment lowered the risk of lesion development in these regions at $4 \mathrm{~h}$. This effect was not noted at the other time points. Further, we found that greater mismatch between predicted outcome using "natural history models" versus "NBO-treatment model" was associated with smaller lesion expansion and in some cases lesion reduction, suggesting these models may be useful in selecting patients most likely to benefit from NBO therapy. We speculate that risk maps that synthesize multiparametric imaging, such as those presented here and by others $[2,32]$, may provide an objective quantitative means for a priori predicting tissue outcome on an individual voxel basis before therapy administration. The algorithms proposed in this study are thus initial steps towards developing tools that will aid the clinician to tailor therapy on an individual patient basis since these models can theoretically be used to determine whether one therapy, e.g. NBO or rt$\mathrm{PA}$, will produce smaller lesion volumes as compared to 
another therapy. This may be useful for neuroradiologists and stroke physicians who are faced with an increasing myriad of imaging modalities [33] aimed at interrogating the state of the ischemic tissue.

Insight into the mechanisms of tissue salvage by these interventions may potentially be gleaned by comparing the coefficients for the different models. For example, we found that MTT plays a greater role in the NBOmodels than in Control-models in predicting which tissue is likely to develop into a lesion at each of the timepoints. ADC and CBV appear to matter less in the NBO during-treatment $4 \mathrm{~h}$ models, suggesting that with NBO, the presence in a voxel of low ADC and low CBV are less likely to result in lesion. This suggests that there may be brief neuroprotection bestowed on at-risk tissue by NBO despite lack of reperfusion (Table 1). In addition, we note that iDWI progressively assumes less weight with Tmax playing a more important role over time for the NBO-model and less importance for the Control-model. We theorize that this is due to preservation of the DWI/PWI mismatch in the early stages of lesion development by NBO therapy, with infarct expansion into the area of abnormality delineated by Tmax maps, which have been speculated to be a reflection of the extent of collateral flow and hence tissue salvage $[23,34,35]$. We also observed greater variability in NBOmodels than in the Control-models. We speculate that this is in part due to greater heterogeneity in patient response to NBO therapy, which are likely mediated by several clinical factors that cannot be captured in neuroimaging, such as age, gender, blood pressure, blood glucose levels, or hematocrit, which have been shown to effect rates of recanalization and hemorrhagic transformation after intravenous or intra-arterial thrombolysis [36]. Although randomization will somewhat compensate for these differences, it may be necessary to explicitly incorporate non-imaging covariates in MRI-based multivariate algorithms in order to improve prediction of tissue outcome in patients given novel intervention.

Tissue-based models have some limitations. For example, the accuracy of predictions depends on the training data [7]. For these models to be applicable to a general stroke population, one would need both larger training data sets and more sophisticated models that incorporate factors that mediate lesion evolution. The imbalance in the rate of reperfusion in our Control group (17\%) compared to NBO group (50\%) could partly explain the differences observed between the model performances. We have shown previously that models predicting outcome of patients after thrombolysis who experience early reperfusion perform poorly, probably due to salvage of tissue that would have otherwise infarcted [7]. Likewise, NBO-therapy may play an indirect role in reperfusion, as evidenced by reports of increased cerebral blood flow and blood volume during NBO in peri-infarct regions [15-17]. However, future studies predicting outcome in dichotomized analyses between patients who reperfuse and those who do not in both groups should be performed to better discriminate the effects of reperfusion on NBO-treated patients. Another important issue before these models can be used in a clinical decision-making setting is reproducibility of model parameters across centers. For example, the coefficients for the Discharge Control-model may differ significantly between studies [7] due to factors such as different onset-to-MRI times, patient inclusion criteria, patient populations, and PWI techniques.

\section{Conclusion}

Our findings indicate that predictive algorithms can be used to estimate risk of lesion development for patients given therapeutic interventions such as NBO. Differences between predicted results for control models and treatment models were found on a volumetric basis (i.e. predicted lesion volume) as well as on a voxel-wise basis (i.e. probability of lesion development) suggesting a therapeutic benefit for NBO at $4 \mathrm{~h}$. Further studies are needed to determine the utility of MRI predictive algorithms in stroke clinical trials, and to confirm the safety and efficacy of NBO in acute ischemic stroke.

\section{Abbreviations}

NBO: Normobaric oxygen therapy; rt-PA: Recombinant tissue plasminogen activator; MRI: Magnetic resonance imaging; DWl: Diffusion-weighted imaging; PWI: Perfusion-weighted imaging; MRA: Magnetic resonance angiography; CBF: Cerebral blood flow; CBV: Cerebral blood volume; MTT: Mean transit time; Tmax: Time to maximum delay; ADC: Apparent diffusion coefficient; T2WI: T2-weighted image; MLV: Measured lesion volume; PLV: Predicted lesion volume; ROI: Region of interest; TP: True positive; FP: False positive; TN: True negative; FN: False negative.

\section{Acknowledgements}

This study was supported in part by NIH grants R01NS051412, R01NS38477, R01NS59775, P50NS051343, and 1R21NS077442; NCRR grants R03 EB008134 and P41RR14075; the MIND Institute; and the Lawrence Award, Hinduja Foundation, NY. The funding agencies had no role in the collection, analysis, and interpretation of data; in the writing of the manuscript; or in the decision to submit the manuscript for publication.

\section{Author details}

${ }^{1}$ Athinoula A. Martinos Center for Biomedical Imaging, Department of Radiology, Massachusetts General Hospital, 149 13th Street, Charlestown MA 02129, USA. ²Department of Neurology, Massachusetts General Hospital, 55 Fruit Street, Boston MA 02114, USA. 3 Department of Radiology, Massachusetts General Hospital, 55 Fruit Street, Boston MA 02114, USA.

\section{Authors' contributions}

Study concept and design: OW, AGS, ABS; Acquisition of data: ABS, TB, LR, MZ, PWS, OW, AGS; Statistical analysis: OW; Analysis and interpretation of data: OW, AGS, ABS; Drafting of the manuscript and critical revisions: OW, AGS, ABS. All authors read and approved the final manuscript.

\section{Competing interests}

O.W. and A.G.S are co-inventors on US Patent 7,020,578, "Method for evaluating novel, stroke treatments using a tissue risk map." The other authors declare that they have no competing interests. 
Received: 1 February 2012 Accepted: 9 March 2012

Published: 9 March 2012

\section{References}

1. Wu O, Koroshetz WJ, Østergaard L, Buonanno FS, Copen WA, Gonzalez RG, Rordorf G, Rosen BR, Schwamm LH, Weisskoff RM, Sorensen AG: Predicting tissue outcome in acute human cerebral ischemia using combined diffusion- and perfusion-weighted MR imaging. Stroke 2001, 32(4):933-942.

2. Jacobs MA, Mitsias P, Soltanian-Zadeh $H$, Santhakumar S, Ghanei A, Hammond R, Peck DJ, Chopp M, Patel S: Multiparametric MRI tissue characterization in clinical stroke with correlation to clinical outcome: Part 2. Stroke 2001, 32(4):950-957.

3. Wu O, Sumii T, Asahi M, Sasamata M, Ostergaard L, Rosen BR, Lo EH, Dijkhuizen RM: Infarct prediction and treatment assessment with MRIbased algorithms in experimental stroke models. J Cereb Blood Flow Metab 2007, 27(1):196-204

4. Jacobs MA, Zhang ZG, Knight RA, Soltanian-Zadeh H, Goussev AV, Peck DJ, Chopp M: A model for multiparametric MRI tissue characterization in experimental cerebral ischemia with histological validation in rat: Part 1. Stroke 2001, 32(4):943-949.

5. Pillekamp F, Grune M, Brinker G, Franke C, Uhlenkuken U, Hoehn M, Hossmann K: Magnetic resonance prediction of outcome after thrombolytic treatment. Magn Reson Imaging 2001, 19(2):143-152.

6. Shen Q, Ren H, Fisher M, Duong TQ: Statistical prediction of tissue fate in acute ischemic brain injury. J Cereb Blood Flow Metab 2005, 25(10):1336-1345.

7. Wu O, Christensen S, Hjort N, Dijkhuizen RM, Kucinski T, Fiehler J, Thomalla G, Rother J, Ostergaard L: Characterizing physiological heterogeneity of infarction risk in acute human ischaemic stroke using MRI. Brain 2006, 129(Pt 9):2384-2393.

8. Singhal $A B$ : Oxygen therapy in stroke: past, present, and future. Int J Stroke 2006, 1(4):191-200.

9. Singhal $A B$ : A review of oxygen therapy in ischemic stroke. Neurol Res 2007, 29(2):173-183.

10. Liu W, Sood R, Chen Q, Sakoglu U, Hendren J, Cetin O, Miyake M, Liu KJ: Normobaric hyperoxia inhibits NADPH oxidase-mediated matrix metalloproteinase-9 induction in cerebral microvessels in experimental stroke. J Neurochem 2008, 107(5):1196-1205

11. Henninger N, Bratane BT, Bastan B, Bouley J, Fisher M: Normobarichyperoxia and delayed tPA treatment in a rat embolic stroke model. J Cereb Blood Flow Metab 2009, 29(1):119-129, Epub 2008 Sep 3.

12. Shin HK, Dunn AK, Jones PB, Boas DA, Lo EH, Moskowitz MA, Ayata C: Normobaric hyperoxia improves cerebral blood flow and oxygenation, and inhibits peri-infarct depolarizations in experimental focal ischaemia. Brain 2007, 130(Pt 6):1631-1642.

13. Henninger N, Bouley J, Nelligan JM, Sicard KM, Fisher M: Normobaric hyperoxia delays perfusion/diffusion mismatch evolution, reduces infarct volume, and differentially affects neuronal cell death pathways after suture middle cerebral artery occlusion in rats. J Cereb Blood Flow Metab 2007, 27(9):1632-1642

14. Veltkamp R, Sun L, Herrmann O, Wolferts G, Hagmann S, Siebing DA, Marti HH, Veltkamp C, Schwaninger M: Oxygen therapy in permanent brain ischemia: potential and limitations. Brain Res 2006, 1107(1):185-191.

15. Liu S, Shi H, Liu W, Furuichi T, Timmins GS, Liu KJ: Interstitial pO2 in ischemic penumbra and core are differentially affected following transient focal cerebral ischemia in rats. J Cereb Blood Flow Metab 2004, 24(3):343-349.

16. Singhal $A B$, Wang $X$, Sumii $T$, Mori $T$, Lo EH: Effects of normobaric hyperoxia in a rat model of focal cerebral ischemia-reperfusion. J Cereb Blood Flow Metab 2002, 22(7):861-868.

17. Singhal AB, Dijkhuizen RM, Rosen BR, Lo EH: Normobaric hyperoxia reduces $M R I$ diffusion abnormalities and infarct size in experimental stroke. Neurology 2002, 58(6):945-952.

18. Singhal $A B$, Benner $T$, Roccatagliata $L$, Koroshetz WJ, Schaefer PW, Lo EH, Buonanno FS, Gonzalez RG, Sorensen AG: A pilot study of normobaric oxygen therapy in acute ischemic stroke. Stroke 2005, 36(4):797-802.

19. Singhal $A B$, Ratai $E$, Benner $T$, Vangel $M$, Lee $V$, Koroshetz WJ, Schaefer PW, Sorensen AG, Gonzalez RG: Magnetic resonance spectroscopy study of oxygen therapy in ischemic stroke. Stroke 2007, 38(10):2851-2854.
20. Gonzalez RG, Hakimelahi R, Schaefer PW, Roccatagliata L, Sorensen AG, Singhal AB: Stability of large diffusion/perfusion mismatch in anterior circulation strokes for 4 or more hours. BMC Neurol 2010, 10:13.

21. Smith EE, Fitzsimmons AL, Nogueira RG, Singhal AB: Spontaneous hyperacute postischemic hemorrhage leading to death. J Neuroimaging 2004, 14(4):361-364.

22. Sled JG, Zijdenbos AP, Evans AC: A nonparametric method for automatic correction of intensity nonuniformity in MRI data. IEEE Trans Med Imaging 1998, 17(1):87-97.

23. Wu O, Ostergaard L, Weisskoff RM, Benner T, Rosen BR, Sorensen AG: Tracer arrival timing-insensitive technique for estimating flow in MR perfusionweighted imaging using singular value decomposition with a blockcirculant deconvolution matrix. Magn Reson Med 2003, 50(1):164-174.

24. Mouridsen K, Christensen S, Gyldensted L, Ostergaard L: Automatic selection of arterial input function using cluster analysis. Magn Reson Med 2006, 55(3):524-531.

25. Jenkinson M, Bannister P, Brady M, Smith S: Improved optimization for the robust and accurate linear registration and motion correction of brain images. Neuroimage 2002, 17(2):825-841.

26. Mazziotta J, Toga A, Evans A, Fox P, Lancaster J, Zilles K, Woods R, Paus T, Simpson G, Pike B, et al: A probabilistic atlas and reference system for the human brain: International Consortium for Brain Mapping (ICBM) Philos Trans R Soc Lond B Bio/ Sci 2001, 356(1412):1293-1322.

27. Collins DL, Neelin P, Peters TM, Evans AC: Automatic 3D intersubject registration of MR volumetric data in standardized Talairach space. J Comput Assist Tomogr 1994, 18(2):192-205.

28. R Development Core Team: R: A Language and Environment for Statistical Computing. Vienna, Austria: R Foundation for Statistical Computing; 2.1 .02005

29. Efron B: The Jackknife, the bootstrap and other resampling plans Philadelphia: SIAM; 1982.

30. Japkowicz N, Stephen S: The class imbalance problem: A systematic study. Intelligent Data Analysis 2002, 6(5):429-450.

31. Chan PH: Reactive oxygen radicals in signaling and damage in the ischemic brain. J Cereb Blood Flow Metab 2001, 21(1):2-14.

32. Rose SE, Chalk JB, Griffin MP, Janke AL, Chen F, McLachan GJ, Peel D, Zelaya FO, Markus HS, Jones DK, et al: MRI based diffusion and perfusion predictive model to estimate stroke evolution. Magn Reson Imaging 2001, 19(8):1043-1053.

33. Butcher KS, Parsons M, MacGregor L, Barber PA, Chalk J, Bladin C, Levi C, Kimber T, Schultz D, Fink J, et al: Refining the perfusion-diffusion mismatch hypothesis. Stroke 2005, 36(6):1153-1159.

34. Christensen S, Wu O, Karstoft H, Hjort N, Butcher K, Davis S, Østergaard L: The physiological significance of the Tmax parameter in bolus tracking MRI (abstr). ISMRM 14th Scientific Meeting Seattle, WA; 2006.

35. Liebeskind DS: Collaterals in acute stroke: beyond the clot. Neuroimaging Clin N Am 2005, 15(3):553-573.

36. Kent DM, Ruthazer R, Selker HP: Are some patients likely to benefit from recombinant tissue-type plasminogen activator for acute ischemic stroke even beyond 3 hours from symptom onset? Stroke 2003, 34(2):464-467.

doi:10.1186/2045-9912-2-5

Cite this article as: Wu et al:: Evaluating effects of normobaric oxygen therapy in acute stroke with MRI-based predictive models. Medical Gas Research 2012 2:5.

\section{Submit your next manuscript to BioMed Central and take full advantage of:}

- Convenient online submission

- Thorough peer review

- No space constraints or color figure charges

- Immediate publication on acceptance

- Inclusion in PubMed, CAS, Scopus and Google Scholar

- Research which is freely available for redistribution 